\title{
Gap Junction Turnover Is Achieved by the Internalization of Small Endocytic Double-Membrane Vesicles
}

\author{
Matthias M. Falk, ${ }^{*}$ Susan M. Baker, ${ }^{*+}$ Anna M. Gumpert, ${ }^{*+}$ \\ Dominique Segretain, ${ }^{\ddagger}$ and Robert W. Buckheit, III*
}

\begin{abstract}
*Department of Biological Sciences, Lehigh University, Bethlehem, PA 18015; and łInstitut National de la Santé et de la Recherche Médicale U895, Université Paris Descartes, 75006 Paris, France
\end{abstract}

Submitted April 9, 2009; Revised May 6, 2009; Accepted May 11, 2009

Monitoring Editor: Sandra L. Schmid

\begin{abstract}
Double-membrane-spanning gap junction (GJ) channels cluster into two-dimensional arrays, termed plaques, to provide direct cell-to-cell communication. GJ plaques often contain circular, channel-free domains $(\sim 0.05-0.5 \mu \mathrm{m}$ in diameter $)$ identified $>30$ y ago and termed nonjunctional membrane (NM) domains. We show, by expressing the GJ protein connexin43 (Cx43) tagged with green fluorescent protein, or the novel photoconvertible fluorescent protein Dendra2, that NM domains appear to be remnants generated by the internalization of small GJ channel clusters that bud over time from central plaque areas. Channel clusters internalized within seconds forming endocytic double-membrane GJ vesicles $(\sim 0.18-0.27 \mu \mathrm{m}$ in diameter) that were degraded by lysosomal pathways. Surprisingly, NM domains were not repopulated by surrounding channels and instead remained mobile, fused with each other, and were expelled at plaque edges. Quantification of internalized, photoconverted Cx43-Dendra2 vesicles indicated a GJ half-life of $2.6 \mathrm{~h}$ that falls within the estimated half-life of 1-5 h reported for GJs. Together with previous publications that revealed continuous accrual of newly synthesized channels along plaque edges and simultaneous removal of channels from plaque centers, our data suggest how the known dynamic channel replenishment of functional GJ plaques can be achieved. Our observations may have implications for the process of endocytic vesicle budding in general.
\end{abstract}

\section{INTRODUCTION}

Gap junctions (GJs) are ubiquitously distributed channels that connect the cytoplasms of two apposing cells, each participating in this connection via a half channel, termed a connexon, to provide direct cell-to-cell communication. Connexons are hexamers of four-pass membrane proteins called connexins (Cxs) (Bruzzone et al., 1996; Kumar and Gilula, 1996). Once trafficked to the plasma membrane (PM), GJ channels form by the head-to-head docking of two connexons, one from each of the apposed membranes. GJ channels then cluster into two-dimensional arrays, or plaques, composed of a few to many thousands of individual channels that vary from tens of square nanometers to several square micrometers in size (Bruzzone et al., 1996; Falk, 2000; Severs et al., 2001). Connexins have been found to have a surprisingly short half-life of only $1-5 \mathrm{~h}$, indicating a rapid GJ channel and Cx protein turnover (Fallon and Goodenough, 1981; Beardslee et al., 1998; Berthoud et al., 2004). Recently,

This article was published online ahead of print in $M B C$ in Press (http: / / www.molbiolcell.org/cgi/doi/10.1091/mbc.E09-04-0288) on May 20, 2009.

Author contributions: M.M.F. designed the research; M.M.F., D. S., A.M.G., and R.W.B. performed the experiments; S.M.B. and A.M.G. analyzed the data; M.M.F. wrote the paper; and S.M.B. edited the manuscript.

+ These authors contributed equally to this work.

Address correspondence to: Matthias M. Falk (mfalk@lehigh.edu).

Abbreviations used: AGJ, annular gap junction; Cx, connexin; GFP, green fluorescent protein; GJ, gap junction; NM, nonjunctional membrane; PM, plasma membrane; wt, wild type. we have shown that entire GJ plaques, or large portions of plaques, can be internalized in a clathrin-dependent endocytic process (Piehl et al., 2007; Baker et al., 2008; Gumpert et al., 2008). GJ internalization generates intracellular doublemembrane GJ vesicles that were previously characterized in the cytoplasm of many different cell types (Ginzberg and Gilula, 1979; Larsen et al., 1979; Mazet et al., 1985; Jordan et al., 2001) and were termed annular gap junctions (AGJs). We further showed that these internalized GJ vesicles could subdivide into smaller vesicles that then were processed by lysosomal degradation pathways (Piehl et al., 2007).

However, internalization of entire, or large portions of GJs cannot account for published observations on plaque assembly and turnover. Gaietta et al. (2002) and we (Lauf et al., 2002) reported that newly synthesized GJ channels accrue along the outer edges of Cx43-based GJ plaques, whereas older channels are simultaneously internalized from their centers, resulting in a continuous, rapid (a few hours) replenishment of the channels of a GJ plaque. Thus, we investigated in depth the dynamic processes that lead to GJ channel internalization and turnover by using high-resolution fluorescence deconvolution and time-lapse microscopy of living cells transiently expressing Cx43 tagged either with green fluorescent protein (GFP) or with a newly available fluorescent protein, Dendra2, that can be photoconverted permanently from green to red fluorescence (Gurskaya et al., 2006; Chudakov et al., 2007), and we combined our studies with ultrastructural analyses of these cells.

\section{MATERIALS AND METHODS}

\section{cDNA Constructs}

Cx43-GFP has been described previously (Falk, 2000). The Cx43 cDNA was excised from pEGFPN1 (Clontech, Mountain View, CA) with EcoRI and 
BamHI and cloned into the respective sites of pDendra2-N (Evrogen, Axxora, San Diego, CA).

\section{Cell Culture, Transfections, and 1,1'-Dioctadecyl-3,3,3',3'- tetramethylindocarbocyanine (DiI) Staining}

Human epithelioid cervix carcinoma cells (HeLa, ATCC CCL 2) were maintained, transfected, stained if appropriate, and prepared for live microscopy as described previously (Falk, 2000; Piehl et al., 2007).

\section{Electron Microscopy}

Freeze-fracture replicas of fetal rat epidermal GJs and ultrathin sections of Cx43-GFP-expressing HeLa cells were prepared as described previously (Risek et al., 1994; Piehl et al., 2007).

\section{Light Microscopy, Photoconversion, Photobleaching, and Image Processing}

Wide-field fluorescence microscopy, time-lapse imaging, and image deconvolution was performed on a DeltaVision Model283 microscope (Applied Precision, Issaquah, WA) as described previously (Falk, 2000; Lauf et al., 2002) On this system, point-spread functions for individual lenses are programmed into the algorithms to achieve superior deconvolution. Confocal microscopy was performed as described previously (Lauf et al., 2002). Channels were merged and image sequences were arranged using Photoshop software (Adobe Systems, San Jose, CA).

Cx43-Dendra2 photoconversion was performed on a 510 META confocal microscope (Carl Zeiss, Jena, Germany) equipped for live-cell imaging (Piehl et al., 2007) as described previously (Chudakov et al., 2007). Preconversion images were acquired using two-line mean averaging in separate channels to avoid bleed-through and were taken at low-illumination settings to prevent unintentional Dendra2 photoconversion (458-nm Argon laser line at 0.05$0.1 \%$ power). HeNe laser power was set so that no fluorescence was detected in the red channel. GJ plaque portions were selected, outlined, and photoconverted using the Bleach Track function of the LSM510 META 3.0 software (488-nm excitation; $15 \%$ power at $50 \%$ power-output of a $30-\mathrm{mW}$ argon laser; 5 iterations). Postconversion images were taken in the red channel only (543 $\mathrm{nm}$ ) to prevent further unintentional photoconversion. Immediately after conversion, a z-stack (12 images spaced $1 \mu \mathrm{m}$ apart) covering the entire cell thickness was acquired, and a three-dimensional (3D)-volume reconstruction was rendered. Plaques were then followed at 2- to 30-s image intervals for up to $1 \mathrm{~h}$. For postconversion images, pinhole diameters were wide opened $(\sim 3-\mu \mathrm{m}$ focal depth) to capture as many internalized vesicles as possible.

Selected areas of PM and GJ plaques (squares and circles) were selected, and GFP and DiI fluorescence was photobleached within 1-2 s by using the Bleach Track function of the LSM510 Meta software (Carl Zeiss) (100\% laser power; 488-, 514-, and 543-nm wavelengths combined; 20 iterations). Cells were then followed at 0.5-s image intervals until DiI fluorescence was completely restored. Regions of interest (ROIs) were selected on the image sequences as shown in Figure 6, and fluorescence intensity within the ROIs was measured and graphed over time. Similarly, fluorescence intensity along lines was plotted over time.

\section{Colocalization of Photoconverted Cx43-Dendra2 Vesicles with Lysosomal Structures}

HeLa cells were seeded onto fibronectin-coated microgrid glass-bottomed dishes (MatTek, Ashland, MA), transfected with Cx43-Dendra2 cDNA, and selected GJ plaques $(n=120)$ were photoconverted as described above. After photoconversion, cells were incubated for $15,30,45,60$, and $120 \mathrm{~min}$ at $37^{\circ} \mathrm{C}$ fixed in ice-cold methanol, and stained with antibodies directed against the lysosomal marker protein lysosome-associated membrane protein (Lamp) 1 (1:50 dilution, mouse monoclonal antibody H4A3; Developmental Studies Hybridoma Bank, University of Iowa, Iowa City, IA), followed by Alexa648conjugated secondary antibodies (Invitrogen, Carlsbad, CA). Cells were imaged with a 510 META confocal microscope (Carl Zeiss). Red and far-red channels were acquired using two-line mean averaging in separate channels to avoid bleed-through. The far-red channel was pseudocolored green, channels were merged, and image sequences were arranged using Photoshop software (Adobe Systems). Yellow puncta visible after merging red and pseudocolored green channels indicates colocalization and lysosomal degradation.

\section{Quantitative and Statistical Image Analyses}

Line-scans were performed using LSM 510 META software (Carl Zeiss). Vesicle diameters on confocal images were measured using the Measuring Tool of the Zeiss software. Mobility of vesicles and NM domains was determined by measuring the distance traveled from image to image using timelapse movie sequences. Measurements were quantified, standard deviations were determined, and data were plotted using Excel (Microsft, Redmond, WA) and Adobe Photoshop software. Statistical analyses were done using Excel's analysis of variance two-tailed Student's $t$ test functions of the data analysis package. In all analyses, a $\mathrm{p}$ value of $<0.05$ was considered significant. Data are expressed as mean \pm SEM.

\section{RESULTS}

\section{GJ Plaques Contain Numerous Nonjunctional Membrane Domains}

To investigate the dynamic processes that lead to the internalization and turnover of GJ channels, we transfected connexin-deficient HeLa cells with Cx43-GFP. Many previous reports have shown that GFP-tagged connexins assemble efficiently into GJs, and form functional GJ channels when expressed in transfected cells in culture, including $\mathrm{HeLa}$ cells (Figure 1A; Jordan et al., 1999; Bukauskas et al., 2000; Falk, 2000). Because HeLa cells are not contact inhibited and may grow on top of each other, GJs can be aligned in two principal orientations: perpendicular to the image plane, providing a view onto their edge; or horizontally, providing a view onto their surface (en face) (Figure 1A). To investigate GJ channel turnover, we imaged GJ plaques $\sim 16 \mathrm{~h}$ after transfection at high light-microscopic magnification $(100 \times$ objective plus $1.5 \times$ auxiliary magnification). Acquired image stacks were deconvolved to further enhance image resolution.

At this magnification, we observed numerous circular, nonfluorescent domains in the fluorescent background of horizontally oriented Cx43-GFP GJ plaques that, based on their lack of fluorescence, presumably were devoid of GJ channels ( 7 in the representative GJ plaque shown in Figure $1 \mathrm{~B}$, arrows); and a comparable number of juxtaposed vesicles that appeared much brighter than the fluorescence emitted from the GJ plaques (6 in Figure 1B, arrowheads). Quantitative analyses of seven GJ plaques showed that between 0.77 and 2.88 (average, $1.82 \pm 0.7$ ) circular nonfluorescent domains and 0.77 and 1.87 (average, $1.32 \pm 0.34$ ) respective vesicles (located $<1.0 \mu \mathrm{m}$ away and in front of the plaque) were detectable per $10 \mu \mathrm{m}^{2}$ of imaged GJ plaque (Figure 1C and Table 1), indicating that the number of nonfluorescent domains and juxtaposed vesicles can vary substantially between plaques. Similar void membrane domains, termed nonjunctional membrane (NM) domains that elicit no signs of associated proteins, can be detected on GJ freeze-fracture replicas (Figure 1D, arrow) and have been reported in numerous ultrastructural GJ investigations (Goodenough and Revel, 1970; Ginzberg and Gilula, 1979; Zampighi et al., 1989; Kamasawa et al., 2006; Rash et al., 2007). Staining living Cx43-GFP-expressing HeLa cells with the lipophilic red dye DiI indicated that both GJ plaques and NM domains contain PM (Figure 1, E and F).

\section{Nonjunctional Membrane Domains Appear to Be Generated by the Internalization of Small GJ Vesicles That Bud from Central Plaque Areas}

To further investigate how vesicles and NM domains were generated, we imaged GJ plaques by time-lapse microscopy at a relatively fast acquisition rate (2- to 6-s intervals). Recordings showed that numerous vesicles appeared to bud from GJ plaques and move away into the cytoplasm, whereas at the same time vesicles appeared to be trafficked to GJs (Supplemental Movie 1). Deconvolving image sequences and significantly enhancing secondary magnification revealed that small vesicles with an apparent diameter of $0.18-0.27 \mu \mathrm{m}(4-6$ pixels in diameter; 1 pixel $=45 \times 45$ nm; Falk, 2000) appeared to be generated repeatedly on the GJ plaque surface (Figure 2, A-C, arrowheads; Supplemental Movies 2-4). Vesicles budded within 1-5 s from the plaques and moved away from the sites of generation. GJ 

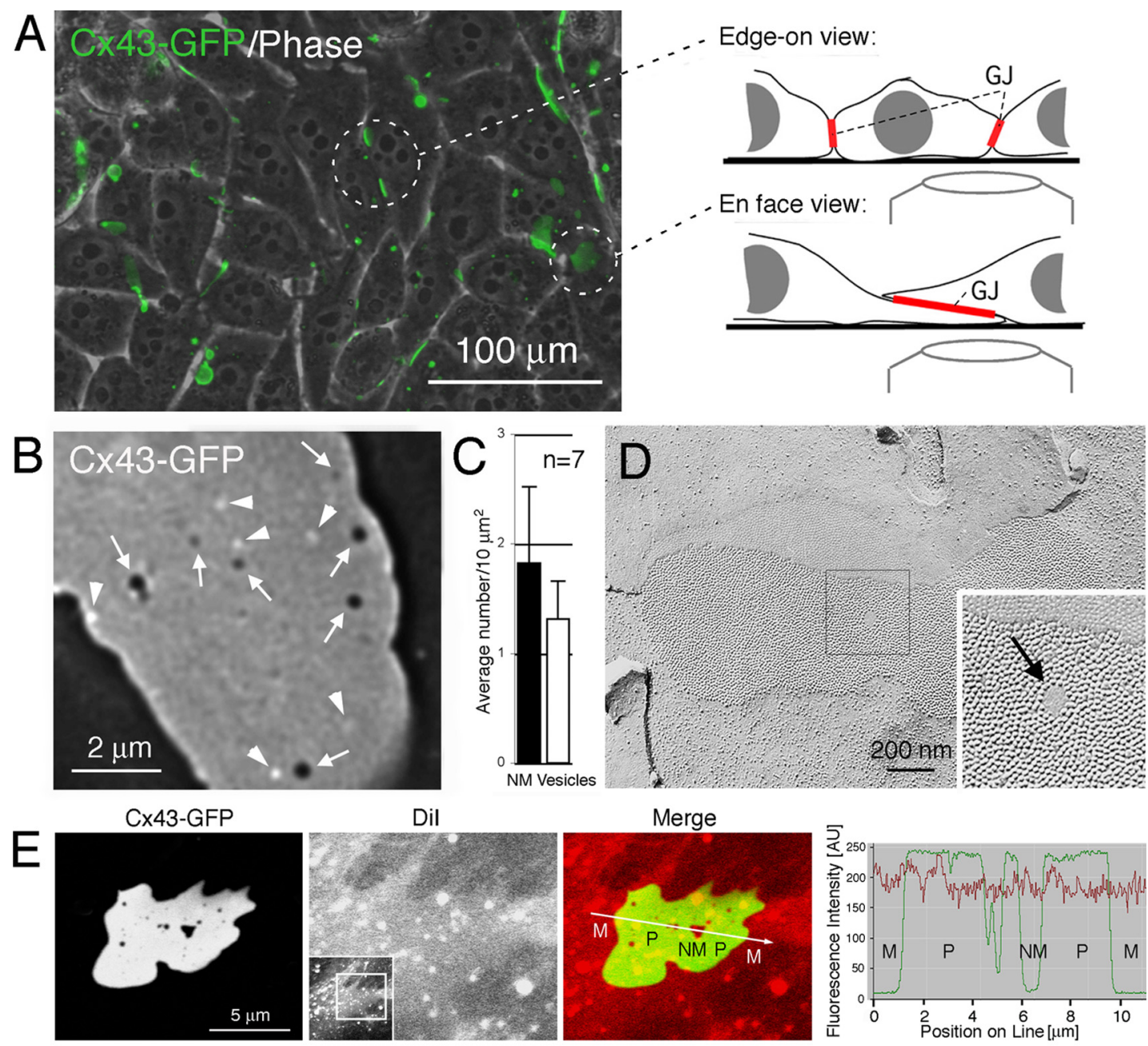

Dil
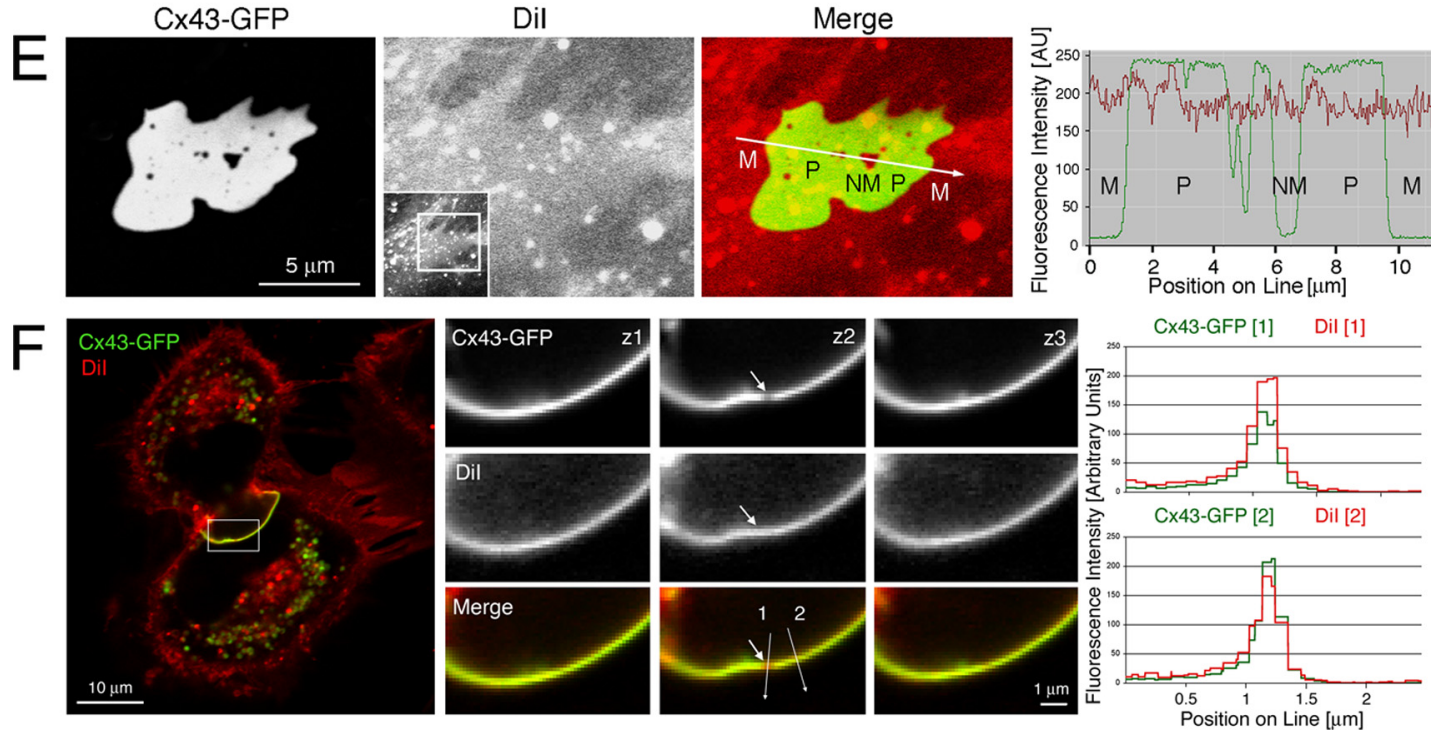

Figure 1. GJ plaques contain circular nonfluorescent domains and juxtaposed bright fluorescent vesicles. (A) HeLa cells expressing Cx43-GFP efficiently assemble GJs in their PMs. GJ plaques can be oriented perpendicular, or horizontally to the image plane (circled). (B) At high light-microscopic resolution, numerous circular, nonfluorescent domains, probably void of GJ channels (dark, arrows), and a comparable number of nearby bright fluorescent vesicles (arrowheads) are detectable in images of horizontally oriented GJ plaques. (C) Quantitative analysis of 7 GJ plaques viewed en face. Large standard deviations indicate that numbers of nonfluorescent domains and juxtaposed vesicles significantly varies between plaques (see Table 1). (D) Circular membrane domains, void of GJ channels, also are detectable by ultrastructural analyses and were termed NM domains or particle-free zones (arrow), as shown, e.g., in a freeze-fracture replica of a GJ plaque derived from fetal rat epidermis. (E and F) GJ plaques and nonjunctional membrane domains contain PM, as indicated by staining with the lipophilic red dye DiI. Cx43-GFP-expressing HeLa cells were stained with DiI ( $5 \mu \mathrm{M}$; $10 \mathrm{~min}) 16 \mathrm{~h}$ after transfection, mounted in a live-cell chamber and imaged immediately after by confocal microscopy. (E) Fluorescence intensities (in arbitrary units) of Cx43-GFP fluorescence (green) and DiI (red) measured along a line traversing PM outside GJs (M), GJ plaques (P), and NM domains of a horizontally oriented GJ plaque. Full-frame image and selected cropped area are shown in the insert. (F) Three sections (z1-z3) spaced 0.2 $\mu \mathrm{m}$ apart through a NM domain (arrow) of a perpendicular-oriented GJ plaque. Quantitative fluorescence intensities of DiI (red) and GFP (green) measured along lines placed through the nonjunctional membrane domain (1), and the GJ plaque (2) are shown on the right. Note the less intense Cx43-GFP fluorescence in the area of the nonjunctional membrane domain in section z2 (top), whereas DiI staining in this area appears at least as intense as in the GJ plaque. 
Table 1. Number of NM domains, respective endocytosed GJ vesicles, and NM domains per $10-\mu \mathrm{m}^{2}$ plaque area calculated for 7 different GJ plaques

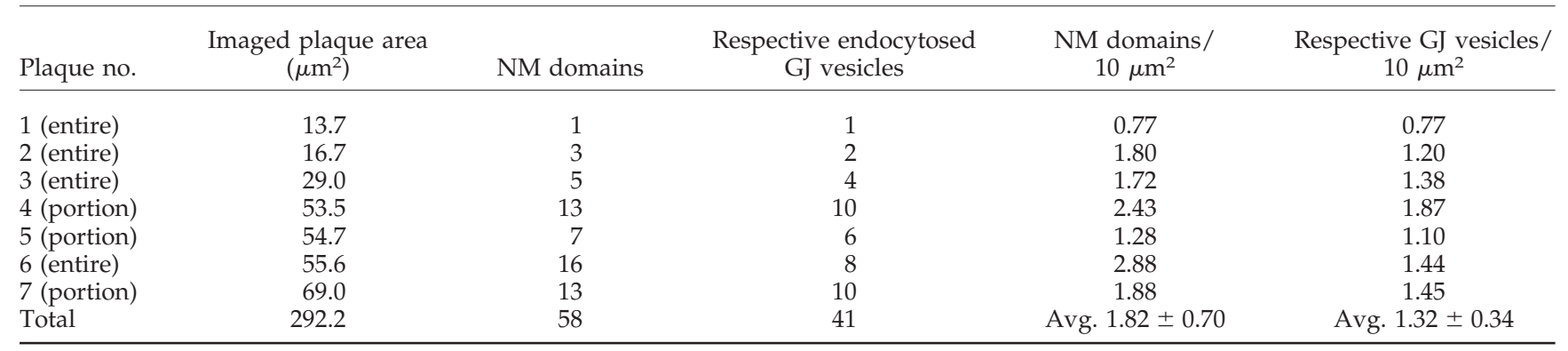

vesicle formation and release appeared to generate small, nonfluorescent circular membrane domains with an apparent approximate diameter of $0.09-0.135 \mu \mathrm{m}$ (2-3 pixels in diameter) that resided for some time within the plaques (Figures 1B, 2A, and 4, A and B, arrows). Vesicle and circular NM domain diameters were determined by counting representative pixels on highly magnified images, and size was calibrated by comparison with images of fluorescent microbeads of known comparable size that were imaged under similar conditions with the identical microscope system (Supplemental Figure S1).

GJ channels appeared to be removed entirely (both connexons), as suggested by the fluorescence intensity of the generated circular NM domains (last time frame of Figure $2 \mathrm{~A}$, arrow) that was comparable with the background fluorescence intensity measured outside GJ plaques (Figure 2D, line-scan of an example plaque; quantified for 15 scanned plaques and internalized vesicles, Figure 2E). Furthermore, internalized GJ vesicles fluoresced twice as intensely as GJ plaques (background fluorescence intensity outside GJ plaques, 7 arbitrary units [AU]; average GJ plaque intensity, $70 \mathrm{AU}$; fluorescence intensity of internalized GJ vesicles, 140 [210 minus 70] AU; Figure 2, D and E), suggesting that GJ channels were internalized as complete double-membranespanning GJ channels. Quantitative analysis of 77 vesicles that budded from nine edge-on viewed GJ plaques (at least $6 /$ plaque) indicated that vesicles preferentially budded into one of two coupled cells; however, with a highly variable bias (58-100\%; Figure $2 \mathrm{~F})$.

\section{Internalized Endocytic GJ Vesicles Move Away from GJ Plaques Deeper into the Cytoplasm}

To further characterize whether the internalization of small channel packets could contribute to the known GJ channel turnover (Gaietta et al., 2002; Lauf et al., 2002), we followed Cx-containing vesicles over time. We identified two significantly different populations of $\mathrm{Cx}$-containing vesicles in the vicinity of GJ plaques (Figure $2 \mathrm{G}$ and Supplemental Movie 5): 1) bright fluorescent vesicles with a normalized fluorescence intensity of $0.62-1.0$ (average $0.88 \pm 0.12 ; \mathrm{n}=26$ ) and an apparent diameter of $0.18-0.27 \mu \mathrm{m}(4-6$ pixels; vesicles $1-3$ circled in Figure $2 \mathrm{G}$, grouped as $>0.15-\mu \mathrm{m}$ diameter in Figure $2 \mathrm{H}$ ) that correlated in fluorescence intensity and size with the previously described GJ vesicles that appeared to bud from central GJ plaque areas (Figure 2, A and D); and 2) dimmer, smaller vesicles with a normalized fluorescence intensity of $0.2-0.6$ (average, $0.40 \pm 0.13 ; n=23 ; p<0.001$ comparing fluorescence intensities of the 2 groups) and an apparent diameter of $0.09-0.135 \mu \mathrm{m}$ (2-3 pixels) that only fluoresced with approximately one half of the intensity of the larger vesicles (vesicles 4-6 circled in Figure 2G, grouped as $<0.15-\mu \mathrm{m}$ diameter in Figure $2 \mathrm{H}$ ). We then determined kinetics of the vesicles on image sequences recorded at 2-s intervals. We found that the larger, brighter GJ vesicles traveled in phases interrupted by periods in which vesicles remained stationary. These vesicles moved away from their release sites on the plaques into the cytoplasm. When moving, vesicles traveled with a speed of 4.0-13.2 $\mu \mathrm{m} / \mathrm{min}$ (average, $7.8 \pm 3.2$; Figure $2 \mathrm{I}$, vesicles $1-3$ ) as calculated for vesicles that traveled in $\mathrm{x}-\mathrm{y}$ and were followed in a single image plane. Smaller, dimmer vesicles traveled uninterrupted and faster with a speed of $12-60 \mu \mathrm{m} / \mathrm{min}$ (average, $30.0 \pm 16.2$; Figure 2I, vesicles $4-6$; $p<0.001$ comparing the velocity of the two groups) as again calculated for vesicles that traveled in $x-y$ and could be followed in a single image plane. These findings correlated with previously published investigations of secretory post-Golgi Cx43 trafficking [2-sample $t$ test comparing the movement of the vesicles published in Lauf et al. (2002), with the dimmer, faster traveling vesicles described here; $p=0.519]$. Vesicle movements also were comparable, although somewhat slower, than the known mobilities of kinesin-1-mediated secretory cargo trafficking $(\sim 48.0 \mu \mathrm{m} / \mathrm{min})$, and the known velocity of myosin-VI-mediated endocytic vesicle trafficking (18.4 $\mu \mathrm{m} / \mathrm{min})$ (Morris et al., 2003). Together, these results support the concept that the slower, brighter vesicles were internalized endocytic GJ vesicles (characterized here), whereas the faster, dimmer vesicles were $\mathrm{Cx}$-containing vesicles trafficking from biosynthetic sites to the surface membrane (characterized in Lauf et al., 2002).

Confocal and ultrastructural examination of Cx43-GFP GJ plaques in fixed HeLa cells revealed different stages of the vesicle generation process (invagination, attached and released vesicles, Figure 3), and the "neck" (arrow) that connects budding vesicles to the GJ plaque (Figure 3, A, E, and F).

\section{Nonjunctional Membrane Domains Can Move, Fuse and Be Expelled at GJ Plaque Edges}

To investigate the fate of the newly generated NM domains, we followed them over time. Recordings showed that they resided in the plaques with unchanged low fluorescence intensity. However, they did not remain stationary, but moved within the plane of the membrane (Figure 4). We further observed that small domains could fuse to form larger NM domains (Figure 4A and Supplemental Movies 6 and 7, 2 or more fuse into 1 domain) and that they eventually were expelled at GJ plaque edges (Figure 4B and Supplemental Movies 8 and 9, small and large domain expelling). Quantitative diameter analyses of 94 circular NM 

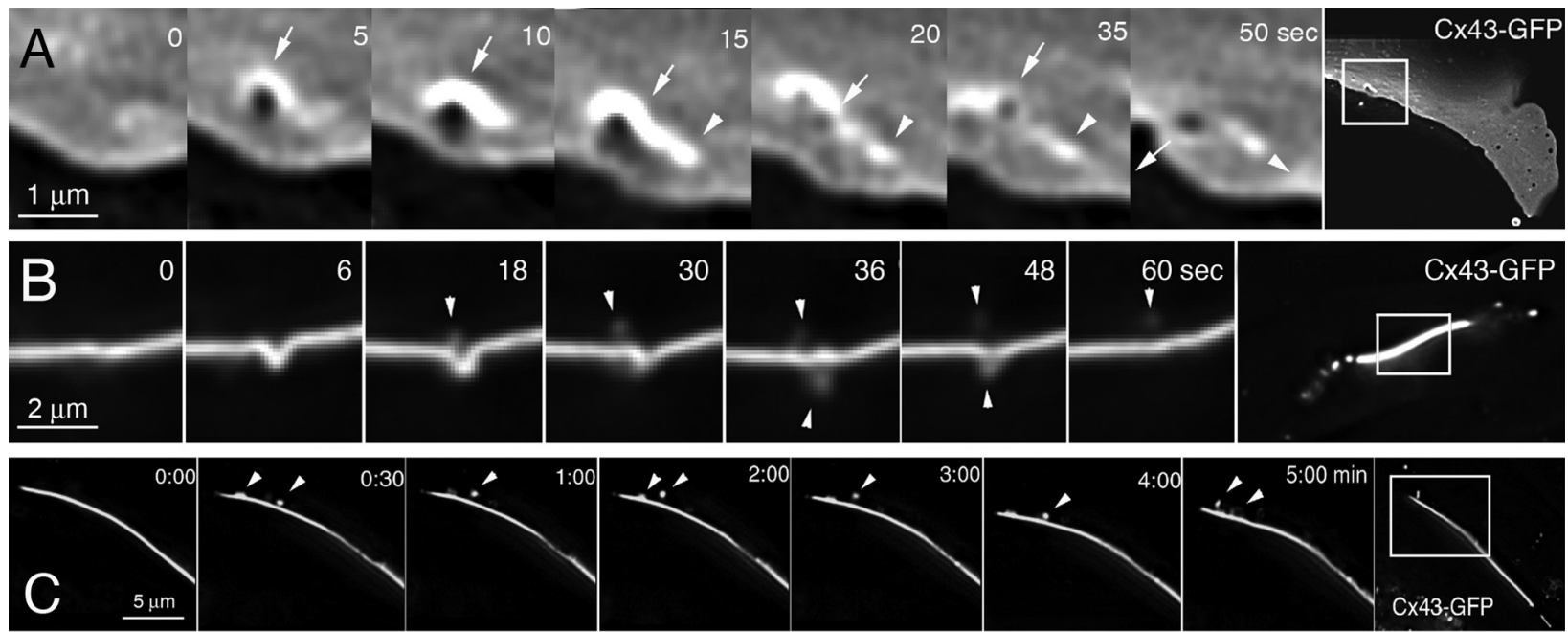
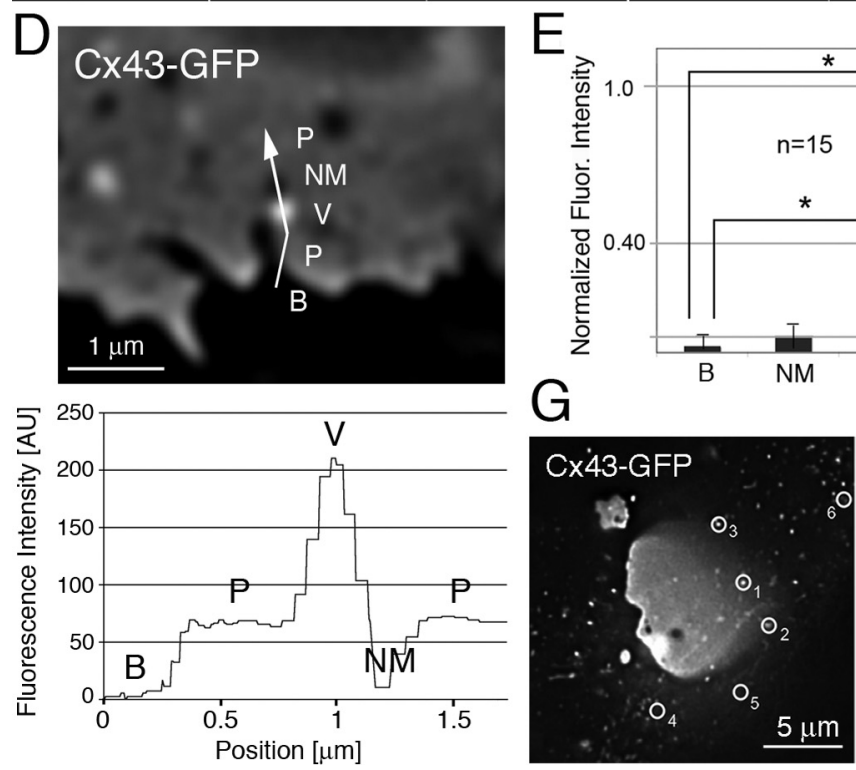

$\mathrm{F}$

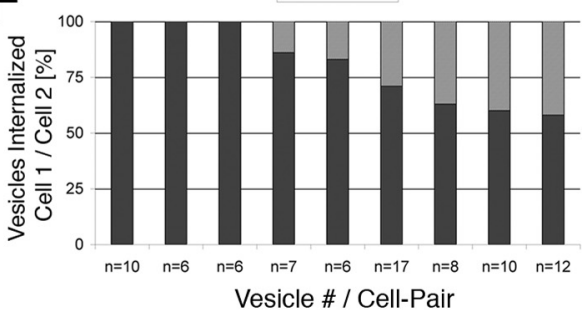

$\mathrm{H}$
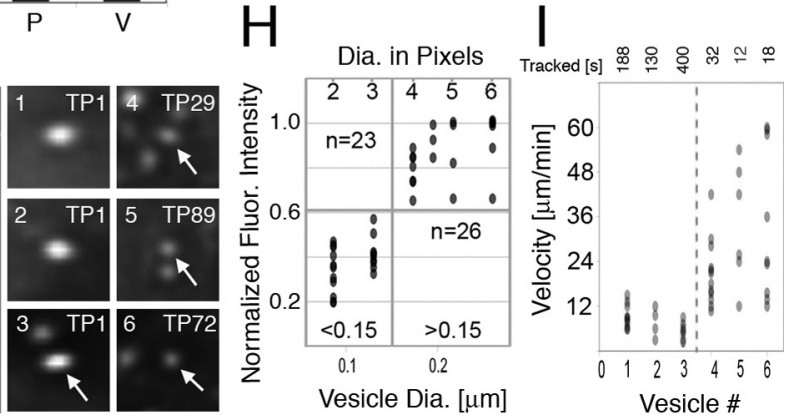

Figure 2. Circular nonfluorescent domains appear to be generated by the internalization of small GJ vesicles that bud continuously from GJ plaques. (A-C) High-resolution time-lapse recordings of Cx43-GFP GJ plaques acquired at relatively fast acquisition rates $(5 \mathrm{~s}$ in $\mathrm{A}$ and $6 \mathrm{~s}$ in B) reveal the internalization of small vesicles (arrowheads) that bud within seconds from central plaque areas. Vesicle budding appears to generate the circular, nonfluorescent membrane domains (dark, arrows) described above that reside for some time within the plaques and that appear to be preceded by an uprising of the plaque indicated by a strong increase of fluorescence around the bud site. NM domain and vesicle move away from their site of generation (indicated by the position of arrow and arrowhead in the 50-s frame). Vesicles in B appear to bud into both coupled cells, and only into one cell in C. Note the similarity between vesicles shown in Figures $1 \mathrm{~B}$ and $2 \mathrm{C}$ and how vesicles in $2 \mathrm{C}$ appear to move along the plaque surface before translocating into the cytoplasm. Vesicles in 2B appear less fluorescent and blurred due to out-of-focus location. Representative magnified frames from Supplemental Movies 2-4 and corresponding full-frame images are shown. (D) Fluorescence intensity (in arbitrary units) measured along a line traversing background area outside a GJ plaque (B), GJ plaque (P), a presumptive internalized GJ vesicle located in front of the plaque (V), and a juxtaposed nonfluorescent membrane domain (NM). The corresponding intensity profile is shown below. (E) Cumulative analyses of 15 comparable line-scans with vesicles located in front of plaques (8) or in front of background (7) $\left({ }^{*} \mathrm{p}<0.001\right)$. Fluorescence of dispersed connexons potentially present in the PM around plaques was not detected. (F) Quantitative analysis of vesicles $(n=77)$ budding from nine edge-on viewed GJ plaques $(\geq 6$ vesicles/plaque) indicates that vesicles preferentially bud into one of two coupled cells; however, with a highly variable bias (58-100\%). (G-I) Fluorescence intensity (G and $\mathrm{H}$ ) and velocity ( $\mathrm{G}$ and I) measurements indicate that two significantly different populations of Cx43-GFP-containing vesicles are present in the vicinity of GJ plaques: 1) small, bright fluorescent vesicles (probably endocytic; see Results) that moved relatively slowly and interrupted by stationary phases (grouped as $>0.15$ in $\mathrm{H}$, vesicles 1-3 in G and I, and additional vesicles on the right in $\mathrm{H}$ ); and 2) even smaller, less fluorescent vesicles (probably secretory; see Results) that moved relatively fast and continuously (grouped as $<0.15$ in H; vesicles $4-6$ in G and I, and additional vesicles on the left in H). The positions of the six vesicles tracked in G and I are circled in the first image (TP1) of the time-lapse sequence and in Supplemental Movie 5. Time points when vesicles first occur are indicated. Dots indicate vesicles in $\mathrm{H}$ and distances traveled between two successive frames in I. Darker dots correspond to the overlapping of multiple observations at each position. $\mathrm{p}$ values comparing vesicle groups in $\mathrm{H}$ and $\mathrm{I}$ were $<0.001$.

domains of seven different GJ plaques revealed that small domains (0.09-0.135- $\mu \mathrm{m}$ apparent diameter; $2-3$ pixels) were $\sim 10$ times more abundant than larger domains (0.4-
0.5- $\mu \mathrm{m}$ apparent diameter; 9-11 pixels, 62 vs. 6 domains) (Figure 4C), supporting our finding that larger NM domains could be generated by the fusion of smaller domains. Quan- 

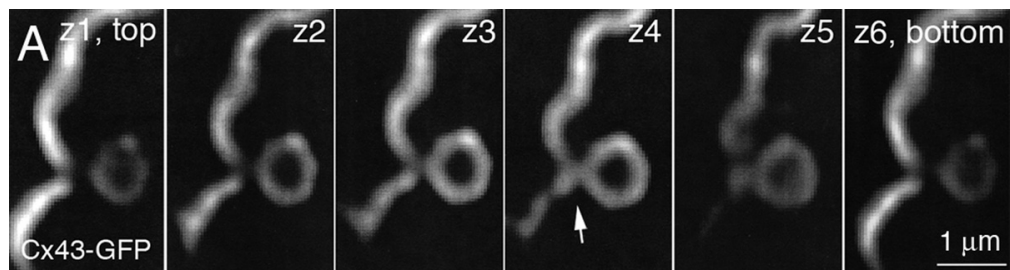

Figure 3. (A) Optical sections spaced $100 \mathrm{~nm}$ apart through a forming vesicle $(\sim 0.7 \mu \mathrm{m}$ in diameter $)$ that buds from a Cx43-GFP GJ plaque in a fixed transfected HeLa cell. Note the "neck" (arrow) that connects the vesicle with the GJ plaque. (B-H) Ultrastructural analyses of thin-sectioned HeLa cells transfected with Cx43GFP. (B) Vesicles of various size and shape are associated with a GJ plaque that is recognizable by its typical penta-laminar staining pattern. (C-H) Double-membrane GJ vesicles (with visible penta-laminar staining pattern in F-H) 100-200 $\mathrm{nm}$ in diameter (arrowheads) at various stages of internalization $(C$ and $D$, plaque invagination; $\mathrm{E}$ and $\mathrm{F}$, attached vesicles; and $\mathrm{G}$ and $\mathrm{H}$, released vesicles). Note the electron dense material visible at the neck of attached vesicles (arrows in E and F).
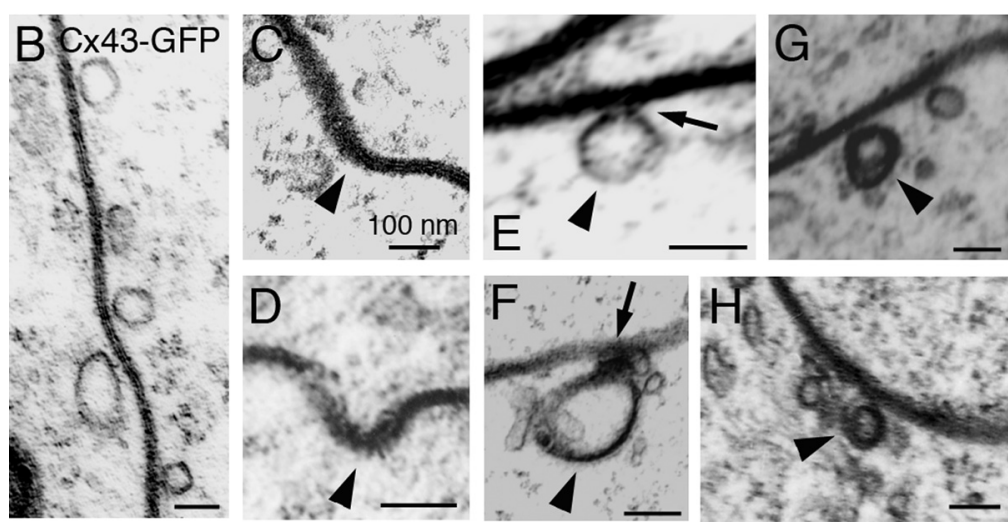

titative movement analyses of circular NM domains revealed that they traveled on average $0.54 \pm 0.05 \mu \mathrm{m} / \mathrm{min}(12$ NM domains; 6 plaques) and that, depending on mobility and plaque size, they generally fused, were expelled, or both from plaques within $30 \mathrm{~min}$ after formation.

\section{Continued Internalization and Degradation of Small GJ Vesicles from Central Plaque Areas Correlates with the Known Rapid GJ Channel Turnover}

To further investigate whether the continued internalization of small GJ channel vesicles could account for the short lifetime of Cxs and the described rapid turnover of GJ channel plaques, we transfected HeLa cells with a photoconvertible Cx43-Dendra2 fusion construct. We permanently photoconverted portions of $\mathrm{Cx} 43$-Dendra2 GJ plaques within 1-2 s from green to red fluorescence by using a $488-\mathrm{nm}$ confocal laser line (spatially restricted to the plaque area outlined in white in example plaques, Figure 5A and Supplemental Figure S2), acquired a Z-scan covering the entire depth of the cells, rendered a 3D-volume reconstruction to verify that no already released $C x$-containing vesicles were accidentally photoconverted, and continued to image the fluorescence signal in the red channel over time. Numerous red fluorescent vesicles were observed to bud from the photoconverted plaque area (at least 10 in the 10-min re- cording time; arrows in Figure 5A and Supplemental Figure S2) and to move away into the cytoplasm. Vesicles internalized from three photoconverted plaques $(n=32)$ were, on average, $0.35 \pm 0.14 \mu \mathrm{m}$ in diameter on raw, nondeconvolved images. This apparent vesicle diameter was comparable with the apparent image size of $0.2-\mu \mathrm{m}$-diameter fluorescent beads of $0.31 \pm 0.014 \mu \mathrm{m}$ on raw, nondeconvolved images (Supplemental Figure S1), suggesting that the true diameter of the released vesicles probably was not $>0.2 \mu \mathrm{m}$. Vesicles moved with an average speed of $2.65 \pm 0.77 \mu \mathrm{m} /$ $\min (\mathrm{n}=10$; stationary and mobile phases combined).

If each internalized GJ vesicle had an average diameter of $0.2 \mu \mathrm{m}$ (as suggested by the vesicle/bead size-correlation described above (Supplemental Figure S1)), 10 vesicles had a combined surface area of $1.26 \mu \mathrm{m}^{2}$. This equates to $\sim 7.56$ $\mu \mathrm{m}^{2}(1.26 \times 6)$ of internalized GJ plaque area per hour, assuming that vesicle internalization continued with the same rate, and all released vesicles were detected. The photoconverted GJ plaque area in Figure $5 \mathrm{~A}$ measured $\sim 8 \mu \mathrm{m}$ in length and $\sim 5 \mu \mathrm{m}$ in depth, equaling $\sim 40 \mu \mathrm{m}^{2}$. Thus, at least $18.9 \%$ of the converted GJ plaque area could have been internalized within $1 \mathrm{~h}$ and one half of the plaque within $\leq 2.6 \mathrm{~h}$

To verify that channel internalization coincided with channel accrual, entire GJ plaques were photoconverted
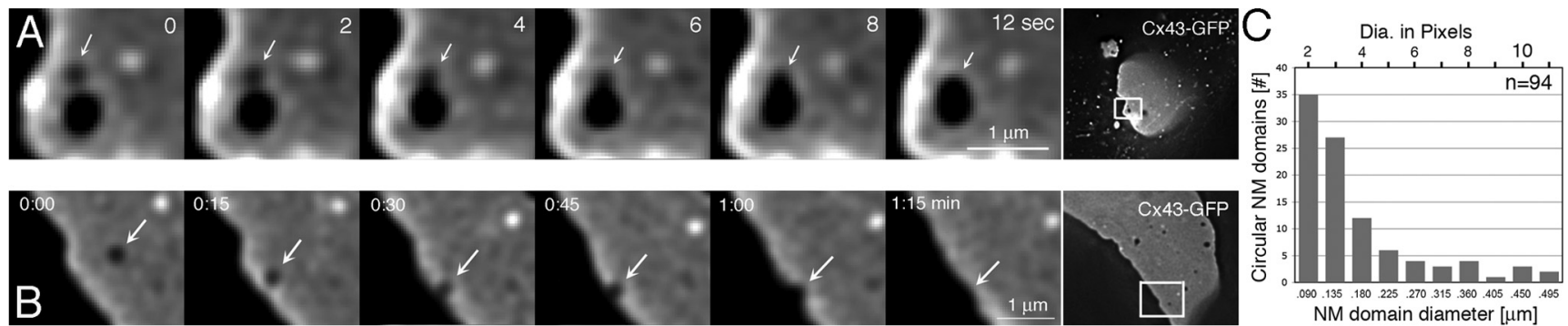

Figure 4. Circular nonfluorescent domains can fuse and are expelled at GJ plaque edges. (A and B) Representative still images from Supplemental Movie 6 showing domain fusion (A) and Supplemental Movie 8 showing domain expelling (B). Magnified frames and full-frame images are shown. Nonfluorescent domains (dark) are labeled with arrows. (C) Quantitative size analysis of 94 nonjunctional membrane domains of seven plaques. 

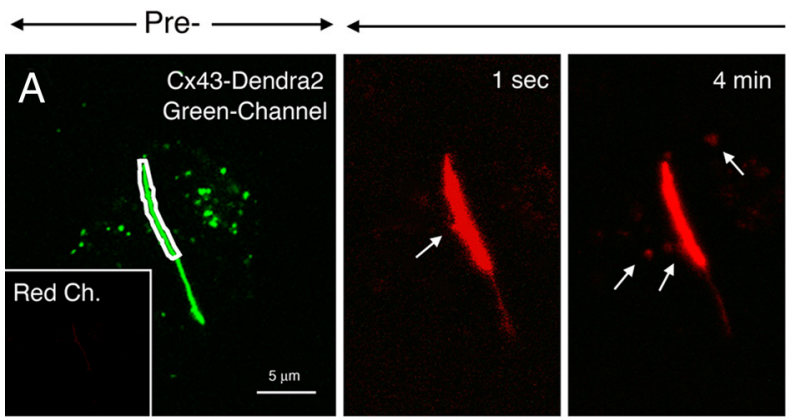

Post-Conversion (Red Channel)
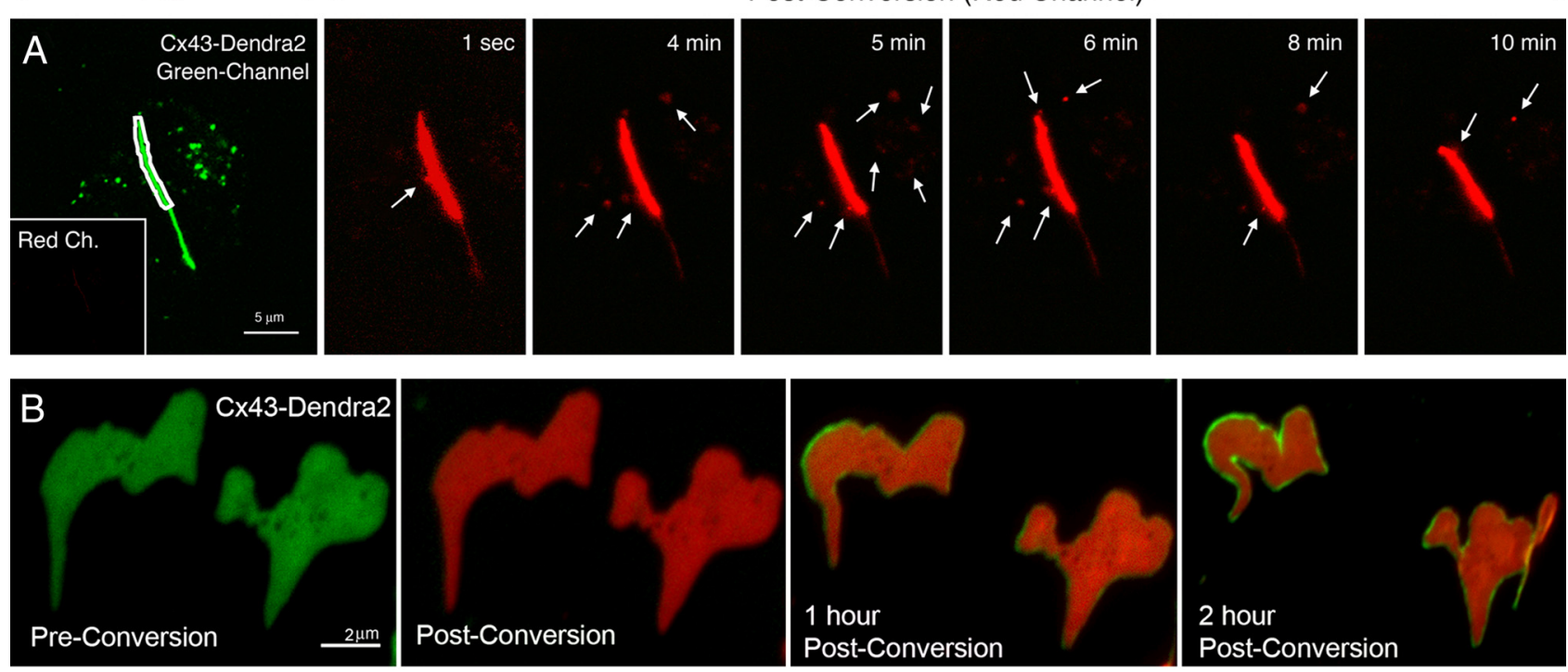

Cx43-Dendra2 converted / Lamp1 (Alexa 648) / Merge
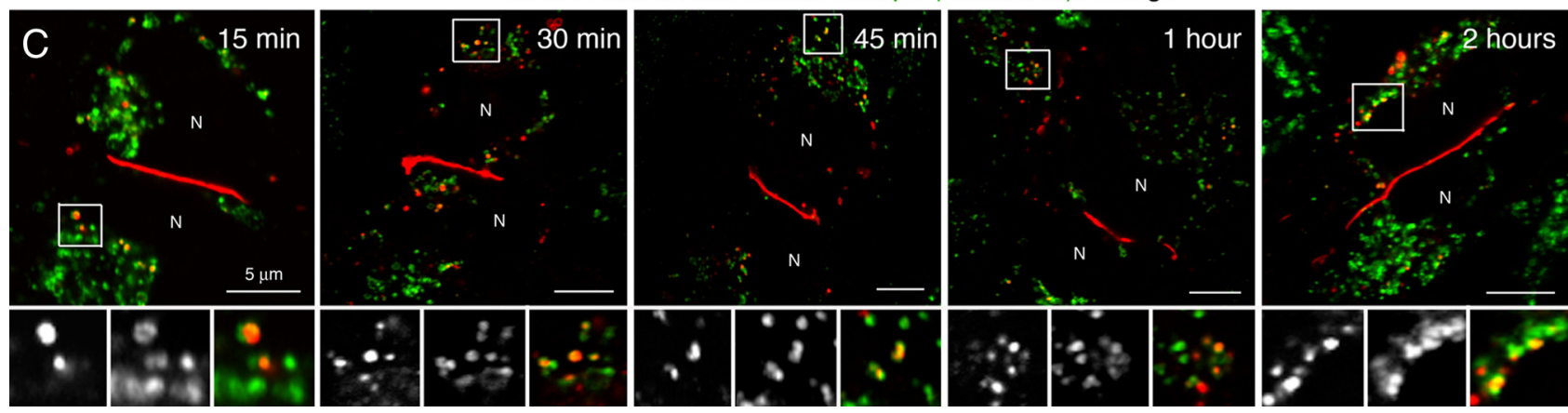

Figure 5. Photoconversion and tracking of Cx43-Dendra2 GJ plaques allows quantification of vesicle internalization, plaque channel turnover, and degradation by lysosomal pathways. (A) Selected areas restricted to GJ plaques assembled from Cx43-Dendra2 (outlined in white on the green channel preconversion image) were permanently photoconverted to red fluorescence and plaques were followed in the red channel over time (postconversion images). At least 10 vesicles (arrows) budded from the photoconverted plaque area within the 10-min time period shown and moved away from the bud sites into the cytoplasm. Preconversion laser power was set low to prevent accidental photoconversion. No fluorescence signal was detectable in the red channel before photoconversion (inset in A; also see Supplemental Figure S2). (B) Entire GJ plaques were photoconverted, and both channels (green and red) were recorded over time. Within $1 \mathrm{~h}$ after conversion, a homogenous green line of GJ channels occurred along the outer plaque edges that widened over time ( $2 \mathrm{~h}$ after conversion), suggesting that newly synthesized, not photoconverted GJ channels accrued along the outer edge of GJs occurring simultaneously with GJ channel internalization. (C) After photoconversion of selected GJ plaques (red), cells were incubated for indicated periods at $37^{\circ} \mathrm{C}$, fixed and stained with antibodies directed against the lysosomal marker protein Lamp1, followed by Alexa648-conjugated secondary antibodies (pseudocolored green) and confocal observation. Numerous photoconverted GJ vesicles (red) colocalized at all time points with Alexa648-labeled Lamp1-positive lysosomal structures as indicated by the yellow overlay color. N, nuclei.

from green to red fluorescence as described above, and both channels (green and red) were recorded over time. Figure 5B shows that within $1 \mathrm{~h}$ after conversion, a homogenous green line of GJ channels formed along the outer plaque edges that widened over time ( $2 \mathrm{~h}$ after conversion), suggesting that newly synthesized, not photoconverted, GJ channels accrued along the outer edge of GJs simultaneously with GJ channel internalization (Figure 5B).

To further investigate the fate of the internalized vesicles, individual GJ plaques were photoconverted from green to red fluorescence and incubated for $15,30,45,60$, and 120 min before fixation and staining with antibodies directed against the lysosomal marker protein Lamp1. Numerous small photoconverted GJ vesicles (red) colocalized at all time points with Alexa648-labeled Lamp1-positive vesicular structures (pseudocolored green) as indicated by the yellow overlay color, suggesting that the internalized GJ vesicles were degraded by lysosomal pathways (Figure 5C).

\section{PM Lipids Can Defuse Freely throughout GJ Plaques Facilitating the Generation of NM Domains}

To address the question from where the extra lipid that fills newly formed NM domains might be recruited, we labeled Cx43-GFP-expressing HeLa cells for short periods with the lipophilic dye DiI, as described previously (Figure 1). Fluorescence of DiI and Cx43-GFP was photobleached in defined areas in the PM and in horizontally and perpendicular oriented GJ plaques (squares in Figure 6, A and B, and a circle in Figure 6C), and fluorescence intensity in photobleached (region 1 in $6, \mathrm{~A}$ and $\mathrm{B}$, and regions 1 and 3 in $\mathrm{C}$ ) and control regions (region 2 in Figure 6, A and B, and regions 2 and 4 in C) was measured on time-lapse images acquired at $0.5-\mathrm{s}$ 

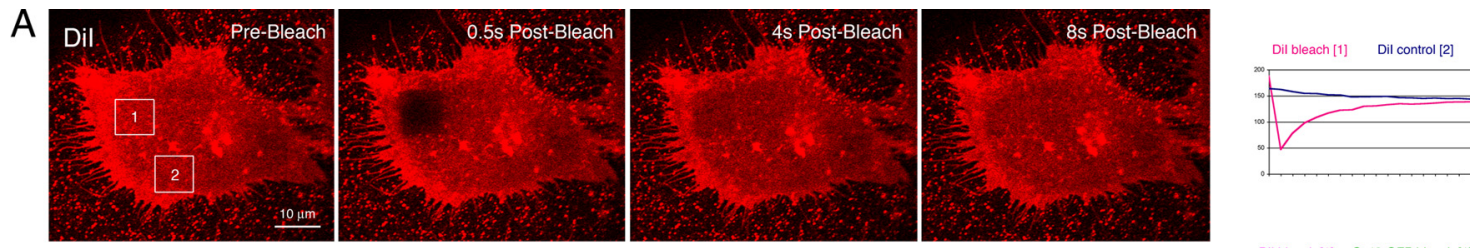

B
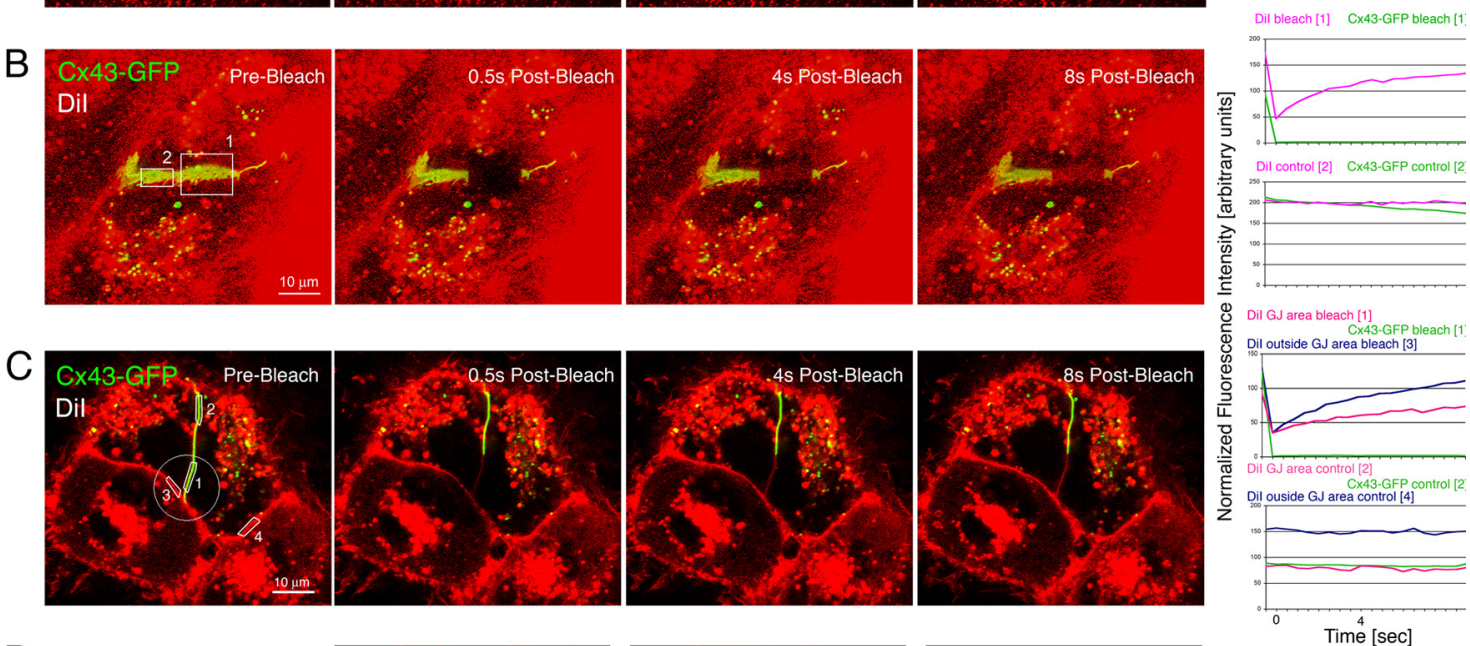

D
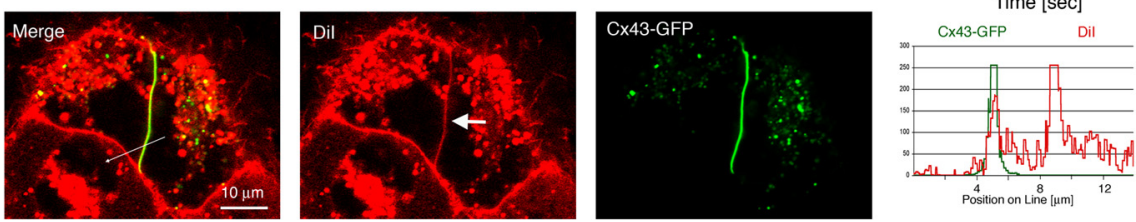

Figure 6. PM lipids can diffuse throughout GJ plaques, facilitating the generation of NM domains. Cx43-GFP-expressing HeLa cells were labeled for $10 \mathrm{~min}$ with the lipophilic dye DiI, as described under Materials and Methods. (A-C) Fluorescence of DiI and Cx43-GFP was photobleached in defined areas in horizontally and perpendicular oriented PM regions, and in GJ plaques (squares in A and B and a circle in C), and fluorescence intensity in photobleached (regions 1 in A and B and regions 1 and 3 in C) and control regions (region 2 in A and $\mathrm{B}$ and regions 2 and 4 in C) was measured on time-lapse images acquired at 0.5-s intervals (plotted on the right). DiI staining in the PM and within GJ plaques recovered within a few seconds correlating with a rapid lateral diffusion of PM lipids within and outside GJ plaques. DiI within GJ plaques recovered slower, as indicated by the shallower slope of the DiI recovery curve (red curve in the recovery graph in D), correlating with a less dynamic mobility of PM lipids within GJ plaques. (D) Consistent with a large portion of the membrane area being occupied by protein channels within GJ plaques, amount of DiI within GJ plaques appeared lower than in PM outside GJ plaques (arrow), as indicated by the height of the DiI fluorescence intensity peaks measured along a line-scan performed before photobleaching, and the higher starting point of the fluorescence recovery curve of DiI-photobleached outside GJs (blue curve in the bleach diagram in C, region 3) compared with the recovery curve of DiI photobleached within the GJ (red curve in the bleach diagram in C, region 1). Cx43-GFP did not show detectable recovery within the 8-s time period, and fluorescence intensity of DiI and Cx43-GFP in nonphotobleached control areas remained stable.

intervals. As expected, DiI staining in the PM recovered within a few seconds $\left(t_{1 / 2}=1.02 \mathrm{~s}\right)$ correlating with the rapid lateral diffusion of PM lipids. Interestingly, DiI within GJ plaques also recovered within a few seconds, indicating that PM lipids within GJs are mobile and can diffuse freely throughout the plaques (Figure 6, B and C); however, mobility appeared somewhat slower as expected, as indicated by the shallower slope of the DiI recovery curve recorded within GJs (red curve in the recovery graph of Figure 6C, region $1 ; t_{1 / 2}=3.05 \mathrm{~s}$ ) compared with the DiI recovery curve recorded outside GJs (blue curve in the recovery graph of Figure $6 \mathrm{C}$, region $3, t_{1 / 2}=2.40 \mathrm{~s}$ ).

Also, consistent with the lower amount of lipid within GJ plaques (a large portion of the membrane is occupied by GJ channels), the amount of DiI within GJ plaques was lower than in PM outside GJ plaques, as indicated by the height of the DiI fluorescence intensity peaks measured along a linescan performed before photobleaching (Figure 6D) and by the higher starting point of the fluorescence recovery curve of DiI photobleached outside GJs (blue curve in the bleach diagram of Figure 6C, region 3) compared with the recovery curve of DiI photobleached within the GJ (red curve in the bleach diagram of Figure 6C, region 1). Cx43-GFP did not show detectable recovery within the 8-s time period, and fluorescence intensity of DiI and Cx43-GFP in nonphotobleached control areas remained stable. Together, these results indicate that the lipid molecules that fill the NM domains can be recruited from surrounding lipids that diffuse within GJ plaques.

\section{DISCUSSION}

Because Cxs are structural membrane proteins and GJ channel function can be regulated via gating, one would expect a long half-life for this class of proteins. However, with the exception of the eye lens, Cxs have relatively short half-lives (reviewed in Laird, 1996; Berthoud et al., 2004; Segretain and Falk, 2004). In cultured cells, 1.5-4 h was determined (Berthoud et al., 2004), and similarly short half-lives of 1.3-5 h were found in liver and heart tissue (Fallon and Goodenough, 


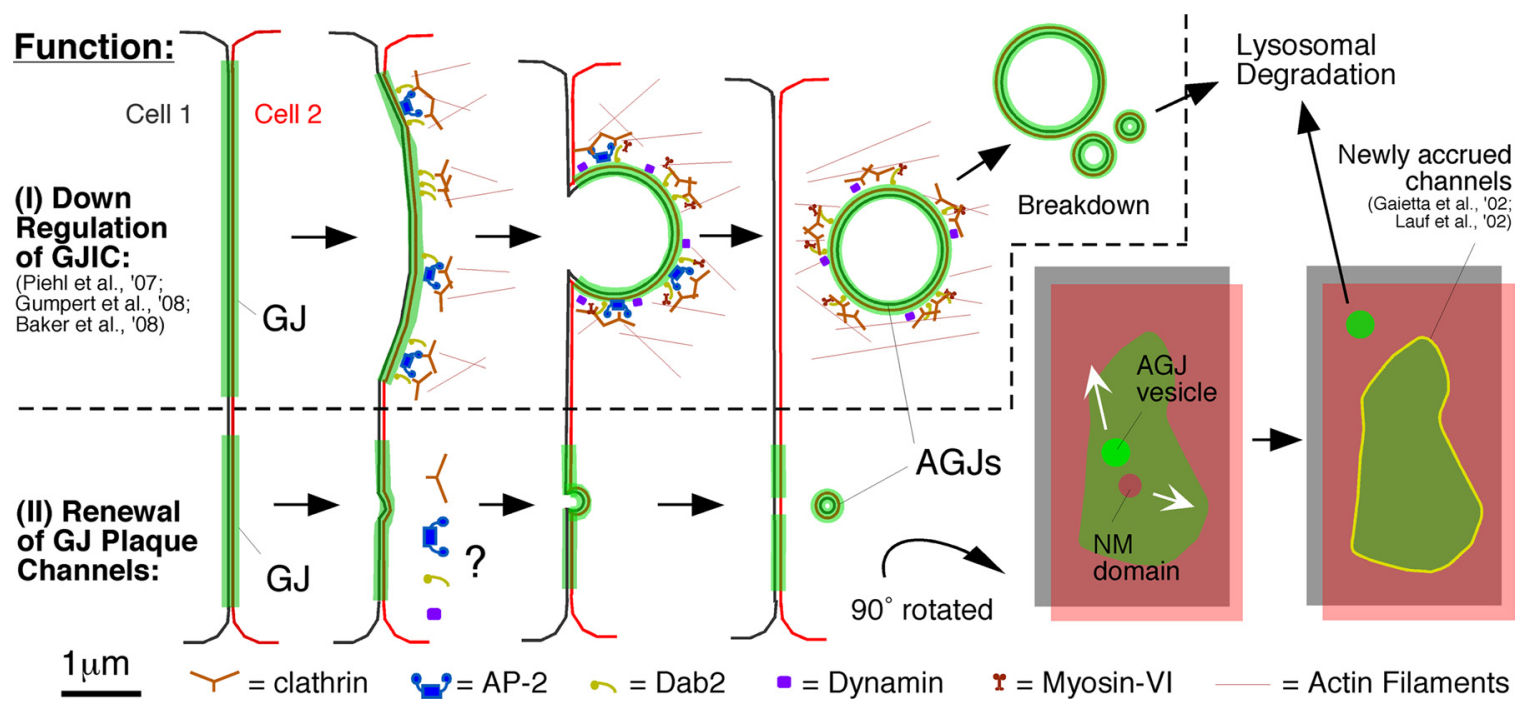

Figure 7. Schematic representation of the pathways that lead to GJ plaque internalization (described in Piehl et al., 2007; Baker et al., 2008; Gumpert et al., 2008) (I) and GJ plaque channel renewal (described here), GJ vesicle formation (AGJ), and GJ vesicle degradation (II). Whether clathrin and clathrin accessory proteins are involved in the internalization of small GJ vesicles described here has not been determined; however, is probably based on our electron microscopy analyses (see Figure 3). Displacement of nonjunctional membrane domains from central plaque areas (NM domain, gray/red) by lateral movement allows the simultaneous accrual of new channels (yellow) along the periphery of GJ plaques (green) without increasing plaque size consistent with previously published observations (Gaietta et al., 2002; Lauf et al., 2002). Clathrin and accessory proteins are shown in patches in accordance to the appearance of clathrin on GJ plaques (Piehl et al., 2007), and the current thinking that clathrin may provide a scaffold for directed actin assembly, facilitating internalization of large structures such as GJs, viruses, and pathogenic bacteria (Pauly and Drubin, 2007; Veiga et al., 2007). The manner in which clathrin and accessory proteins are drawn remains speculative.

1981; Beardslee et al., 1998). By successively staining tetracysteine-tagged Cx43 green and red (Gaietta et al., 2002), or permanently photobleaching portions of GFP-tagged Cx43 GJ plaques (Lauf et al., 2002), it was demonstrated that the channels within a GJ plaque are dynamically exchanged, with newly synthesized channels being accrued to outer plaque edges, whereas older channels were simultaneously removed from central plaque areas (Gaietta et al., 2002; Lauf et al., 2002). Dynamic turnover of yellow fluorescent proteintagged Cx43 GJ plaques, however, with a questionable halflife of only $2.8 \mathrm{~min}$, also has been reported (Shaw et al., 2007). Recently, we reported that entire GJs, or large portions of GJs can be internalized in a clathrin-mediated endocytic process that involves the coat protein clathrin, the clathrin-adaptors AP-2 and Dab2, the GTPase dynamin, and the retrograde actin motor myosin-VI (Piehl et al., 2007; Gumpert et al., 2008) (Figure 7, top). However, internalization of GJs cannot account for the continuous turnover of channels in plaques (Jordan et al., 2001; Gaietta et al., 2002; Lauf et al., 2002).

Following individual Cx43-GJ plaques at high spatial and temporal resolution revealed that over time, multiple small domains of the plaques were internalized within seconds as vesicles (with an apparent diameter of $0.18-0.27 \mu \mathrm{m}$ ) from central regions of plaques (Figures 2 and 5). Vesicle internalization apparently resulted in the formation of circular plasma membrane lipid domains $(0.09-0.135-\mu \mathrm{m}$ apparent diameter) that resided within the plaques. These domains exhibited low fluorescence intensity, comparable with PM regions outside GJ plaques (Figure 2, D and E), suggesting that they were void of GJ channels or connexons. Similar domains are also visible in freeze-fracture replicas of GJ plaques (Figure 1D) and were termed particle-free or nonjunctional membranes (Goodenough and Revel, 1970; Ginzberg and Gilula, 1979; Zampighi et al., 1989; Kamasawa et al., 2006; Rash et al., 2007). To obtain valuable size measurements, we purposely oversampled our images $(1500 \times$ combined magnification) to decrease the image area corresponding to a single pixel of the recording device as much as possible $(45 \times 45 \mathrm{~nm})$, deconvolved the image sequences to further enhance resolution, and calibrated our size measurements with fluorescent microspheres (Supplemental Figure S1). However, apparent diameters of internalized GJ vesicles and of newly generated NM domains are at the limit of light microscopic resolution, and thus size measurements might not be absolutely accurate. Also, our recordings in general did not capture all steps of the vesicle release process, indicating that this process likely lasts only a fraction of a second.

Interestingly, different GJ morphologies have been distinguished by freeze-fracture replica immunogold labeling (FRIL). Besides typical "plaque" type GJs with densely arranged hexagonal or irregular arrays of GJ channels, "string" GJs (rows of channels) and "reticular" GJs with morphologies between plaque and string GJs have been characterized, and it has been suggested that reticular GJs may represent transitional states between plaque and string GJs (Kamasawa et al., 2006; Rash et al., 2007). However, based on our findings, it is tempting to speculate that reticular GJs also could represent small GJ plaques with their central channel portions removed by vesicle internalization as observed in our study.

Fluorescence intensity of internalized GJ vesicles measured over background was twice as bright as the average fluorescence intensity of GJ plaques (Figure 2, D and E), which correlates with the number of GFP layers in plaques and GJ vesicles (2 layers of GFP in GJ plaques vs. 4 layers in GJ vesicles; see Falk, 2000), and suggests that GJ channels were internalized as complete, double-membrane-spanning GJ channels. This hypothesis is supported by our ultrastruc- 
tural analyses that show vesicles with penta-laminar staining typical for GJs attached to GJ plaques (Figure 3), and by FRIL images of GJ plaques in goldfish Mauthner cells that contain circular depressions and elevations, presumably representing budding vesicles with $\mathrm{P}$ - to E-face fracture transitions within the buds (Rash and Pereda, unpublished data). Internalization of double-membrane-spanning GJ channels also was observed in the internalization of entire GJ plaques and large plaque fragments (Jordan et al., 2001; Piehl et al., 2007), and this also led to the formation of cytoplasmic double-membrane GJ vesicles.

To test whether the continuous internalization of small GJ channel packets could account for the known rapid $C x$ and GJ channel turnover, we expressed a Dendra2-tagged Cx43 fusion construct. Dendra2 is a novel green fluorescent protein that can be photoconverted permanently into a red fluorescent form upon excitation with UV, or intense blue light (Gurskaya et al., 2006; Chudakov et al., 2007). We converted portions of Cx43-Dendra2 GJs from green to red fluorescence and continued to image the converted plaques over time. We observed numerous red fluorescent vesicles to bud continuously from the plaques and to move away into the cytoplasm (Figure 5A and Supplemental Figure S2). Z-scans and 3D-volume reconstructions performed immediately after photoconversion indicated that red vesicles budded from the plaques after conversion and were not previously present cytoplasmic vesicles that were photoconverted as well. When green and red channels were recorded for longer times (1-2 h), a homogenous green line of GJ channels was then observed along the outer plaque edges that widened over time, suggesting that newly synthesized GJ channels accrued along the outer edge of GJs simultaneously with GJ channel internalization (Figure 5B). Channel accrual along the outer edge of GJ plaques and dynamics of channel accrual conformed to previous observations using fluorescence recovery after photobleaching (Lauf et al., 2002), and successive FlAsH/ReAsH staining (Gaietta et al., 2002) techniques. Calculating numbers of internalized vesicles and surface areas suggested a half-life of $2.6 \mathrm{~h}\left(\sim 7.5 \mu \mathrm{m}^{2}\right.$ of a $40-\mu \mathrm{m}^{2}$ photoconverted GJ plaque area was internalized in $1 \mathrm{~h}$ ) that falls within the estimated half-life of $1-5 \mathrm{~h}$ reported previously for Cxs and GJs (Fallon and Goodenough, 1981; Beardslee et al., 1998; Berthoud et al., 2004). It is possible that the photoconversion process may have affected vesicle internalization rates; however, photoconversion took only 1-2 s; was restricted to a small portion of the coupled cells; and cells were not observed to round up, even when followed for longer periods (up to 2 h, Figure 5B).

Interestingly, although GJ plaques as well as NM domains were found to contain PM (based on DiI staining, Figure 1, E and F), surrounding GJ channels appeared not to repopulate the newly generated channel-free membrane domains (Figures 1B, 2A, and 4, A and B). This suggests that either the lipid composition of the NM domains differs from the channel portion of the plaque preventing repopulation, or more likely that channels within a GJ plaque retain a characteristic distance to each other, probably based on electrostatic interactions, hydrophobic interactions, or both. Most likely, these same forces also account for the circular nature of the NM domains. Our observation is consistent with the reported finding that channel turnover within a GJ plaque can occur without an increase in plaque size (Gaietta et al., 2002; Lauf et al., 2002). If GJ channels would increase their center-tocenter spacing to repopulate the newly generated channelfree domains, central plaque areas should contain increasingly fewer channels over time; however, such a gradual channel distribution is not visible on freeze-fracture replicas of GJ plaques. GJ channels have been described to pack with different densities; however, this is likely to either be due to the presence of two different GJ channel types (assembled from different $\mathrm{Cx}$ isoforms) that have different packing characteristics (Risek et al., 1994), different developmental and cellular differentiation stages (Schuetze and Goodenough, 1982; Kamasawa et al., 2006; Rash et al., 2007), or different dynamic stages of GJ channel plaque assembly (Johnson et al., 1974; Rash et al., 2007).

To achieve GJ channel turnover without repopulating NM domains or increasing plaque size requires that newly generated NM domains be eliminated from GJ plaques. Indeed, we observed that smaller NM domains could collide and fuse with each other to form larger domains and that they moved throughout plaques before being expelled at plaque edges (Figure 4, A and B). This observation was further supported by a quantitative diameter analysis that showed that small circular domains $(0.09-0.135-\mu \mathrm{m}$ apparent diameter, the size generated by a single vesicle release) were $\sim 10$ times more abundant than circular NM domains with a diameter of $0.4-0.5 \mu \mathrm{m}$ (62 vs. 6, Figure 4C). Also, larger NM domains often were located closer to plaque edges (Figures $1 \mathrm{~B}$ and 4A). Quantitative movement analyses of NM domains demonstrated that they moved with an average speed of $0.54 \pm 0.05 \mu \mathrm{m} / \mathrm{min}(\mathrm{n}=12)$, which supports the concept that they can be expelled from plaques in a time frame that is well below the 1- to 5-h half-life of GJ plaque channels. Obviously, movement of NM domains throughout plaques requires that GJ channels can exchange positions and move with respect to each other. Movements of NM domains might be driven by cell locomotion, cell shape changes, cytoskeletal dynamics that cause directional movements of PM lipids or a combination.

The formation of circular membrane domains upon GJ vesicle budding is a remarkable observation that provides some information about the vesicle release process itself, and it is tempting to speculate that our observation may have implications for other vesicle budding processes as well. Expressing fluorescent protein-tagged Cxs permits labeling of large regions of the PM much more stably than lipid labeling, or labeling of more dynamic, dispersed membrane proteins would permit and thus may allow to resolve specific stages and features of the vesicle release process in greater detail. One important question raised by this observation addresses the lipid balance of GJ plaques. If vesicle internalization generates NM domains, where does the extra lipid required to fill these domains might come from? To address this question, we photobleached DiI within GJ plaques. DiI recovered within a few seconds, indicating that PM lipids within GJs are mobile and can diffuse freely throughout the plaques and thus can fill the NM domains (Figure 6). Interestingly, DiI mobility appeared somewhat slower within GJ plaques compared with PM DiI mobility outside GJs, suggesting that, as expected due the dense packing of the GJ channels, lipid mobility inside GJs was reduced compared with PM lipid mobility outside GJs.

Together with previously published findings (Gaietta et al., 2002; Lauf et al., 2002; Piehl et al., 2007; Gumpert et al., 2008), our reports indicate that GJs are turned over in at least two distinct manners: 1) A relatively slow (20- to 60-min range) internalization of large portions or entire GJ plaques that generates large cytoplasmic GJ vesicles $(0.5-5 \mu \mathrm{m}$ in diameter) that slowly (minutes to hours) breakdown and translocate deeper into the cell for degradation (Piehl et al., 2007); and 2) a continuous and fast (a few seconds) internalization of small $(\sim 0.18-0.27-\mu \mathrm{m}$-diameter) GJ vesicles that bud from central regions of the plaques and translocate 
much more rapidly (within minutes) into the cell for degradation (reported here) (Figure 7). Continuous internalization of small GJ vesicles described here correlates with the known rapid turnover of GJ plaque channels (Gaietta et al., 2002; Lauf et al., 2002). Probably, the two modes of GJ degradation will serve different functions: 1) significant down-regulation of gap junction mediated intercellular communication and 2) continuous replenishment of "spent" GJ plaque channels (Figure 7). Whether the internalization of small GJ vesicles also involves the endocytic clathrin machinery as has been described for the internalization of GJ plaques (Piehl et al., 2007; Gumpert et al., 2008) needs to be determined. However, clathrin-mediated endocytosis of small GJ vesicles appears even more plausible, because observed vesicle size and release kinetics correlate well with classical clathrin-mediated internalization events at the PM.

\section{ACKNOWLEDGMENTS}

We thank George Klier (deceased) for the freeze-fracture image of an epidermal GJ plaque, Jean-Pierre Denizot (Unité de Neurosciences Intégratives et Computationnelles, Centre National de la Recherche Scientifique, Gif sur Yvette, France) for the preparation of ultrathin sections, and William B. Kiosses (Imaging Facility, The Scripps Research Institute, La Jolla, CA) for imaging fluorescent microbeads. This work was supported by National Institutes of Health National Institute of General Medical Sciences grant GM55725 (to M.M.F.) and Lehigh University.

\section{REFERENCES}

Baker, S. M., Kim, N., Gumpert, A. M., Segretain, D., and Falk, M. M. (2008). Acute internalization of gap junctions in vascular endothelial cells in response to inflammatory mediator-induced G-protein coupled receptor activation. FEBS Lett. 582, 4039-4046.

Beardslee, M. A., Laing, J. G., Beyer, E. C., and Saffitz, J. E. (1998). Rapid turnover of connexin43 in the adult rat heart. Circ. Res. 83, 629-635.

Berthoud, V. M., Minogue, P. J., Laing, J. G., and Beyer, E. C. (2004). Pathways for degradation of connexins and gap junctions. Cardiovasc. Res. 62, 256-267.

Bruzzone, R., White, T. W., and Paul, D. L. (1996). Connections with connexins: the molecular basis of direct intercellular signaling. Eur. J. Biochem. 238, $1-27$.

Bukauskas, F. F., Jordan, K., Bukauskiene, A., Bennett, M. V., Lampe, P. D. Laird, D. W., and Verselis, V. K. (2000). Clustering of connexin 43-enhanced green fluorescent protein gap junction channels and functional coupling in living cells. Proc. Natl. Acad. Sci. USA 97, 2556-2561.

Chudakov, D. M., Lukyanov, S., and Lukyanov, K. A. (2007). Tracking intracellular protein movements using photoswitchable fluorescent proteins PSCFP2 and Dendra2. Nat. Protoc. 2, 2024-2032.

Falk, M. M. (2000). Connexin-specific distribution within gap junctions revealed in living cells. J. Cell Sci. 113, 4109-4120.

Fallon, R. F., and Goodenough, D. A. (1981). Five-hour half-life of mouse liver gap-junction protein. J. Cell Biol. 90, 521-526.

Gaietta, G., Deerinck, T. J., Adams, S. R., Bouwer, J., Tour, O., Laird, D. W. Sosinsky, G. E., Tsien, R. Y., and Ellisman, M. H. (2002). Multicolor and electron microscopic imaging of connexin trafficking. Science 296, 503-507.

Ginzberg, R. D., and Gilula, N. B. (1979). Modulation of cell junctions during differentiation of the chicken otocyst sensory epithelium. Dev. Biol. 68, 110129.

Goodenough, D. A., and Revel, J. P. (1970). A fine structural analysis of intercellular junctions in the mouse liver. J. Cell Biol. 45, 272-290.

Gumpert, A. M., Varco, J. S., Baker, S. M., Piehl, M., and Falk, M. M. (2008). Double-membrane gap junction internalization requires the clathrin-mediated endocytic machinery. FEBS Let. 582, 2887-2892.
Gurskaya, N. G., Verkhusha, V. V., Shcheglov, A. S., Staroverov, D. B., Chepurnykh, T. V., Fradkov, A. F., Lukyanov, S., and Lukyanov, K. A. (2006) Engineering of a monomeric green-to-red photoactivatable fluorescent protein induced by blue light. Nat. Biotechnol. 24, 461-465.

Johnson, R., Hammer, M., Sheridan, J., and Revel, J. P. (1974). Gap junction formation between reaggregated Novikoff hepatoma cells. Proc. Natl. Acad. Sci. USA $71,4536-4540$.

Jordan, K., Chodock, R., Hand, A. R., and Laird, D. W. (2001). The origin of annular junctions: a mechanism of gap junction internalization. J. Cell Sci. 114 763-773.

Jordan, K., Solan, J. L., Dominguez, M., Sia, M., Hand, A., Lampe, P. D., and Laird, D. W. (1999). Trafficking, assembly, and function of a connexin43-green fluorescent protein chimera in live mammalian cells. Mol. Biol. Cell 10, 2033-2050.

Kamasawa, N., et al. (2006). Abundance and ultrastructural diversity of neuronal gap junctions in the OFF and ON sublaminae of the inner plexiform layer of rat and mouse retina. Neuroscience 142, 1093-1117.

Kumar, N. M., and Gilula, N. B. (1996). The gap junction communication channel. Cell 84, 381-388

Laird, D. W. (1996). The life cycle of a connexin: gap junction formation, removal, and degradation. J. Bioenerg. Biomembr. 28, 311-318.

Larsen, W. J., Tung, H. N., Murray, S. A., and Swenson, C. A. (1979). Evidence for the participation of actin microfilaments and bristle coats in the internalization of gap junction membrane. J. Cell Biol. 83, 576-587.

Lauf, U., Giepmans, B. N., Lopez, P., Braconnot, S., Chen, S. C., and Falk, M. M. (2002). Dynamic trafficking and delivery of connexons to the plasma membrane and accretion to gap junctions in living cells. Proc. Natl. Acad. Sci. USA 99, 10446-10451.

Mazet, F., Wittenberg, B. A., and Spray, D. C. (1985). Fate of intercellular junctions in isolated adult rat cardiac cells. Circ. Res. 56, 195-204.

Morris, C. A., Wells, A. L., Yang, Z., Chen, L. Q., Baldacchino, C. V., and Sweeney, H. L. (2003). Calcium functionally uncouples the heads of myosin VI. J. Biol. Chem. 278, 23324-23330.

Pauly, B. S., and Drubin, D. G. (2007). Clathrin: an amazing multifunctional dreamcoat? Cell Host Microbe 2, 288-290.

Piehl, M., Lehmann, C., Gumpert, A., Denizot, J. P., Segretain, D., and Falk, M. M. (2007). Internalization of large double-membrane intercellular vesicles by a clathrin-dependent endocytic process. Mol. Biol. Cell 18, 337-347.

Rash, J. E., Olson, C. O., Pouliot, W. A., Davidson, K. G., Yasumura, T., Furman, C. S., Royer, S., Kamasawa, N., Nagy, J. I., and Dudek, F. E. (2007). Connexin36 vs. connexin32, "miniature" neuronal gap junctions, and limited electrotonic coupling in rodent suprachiasmatic nucleus. Neuroscience 149 $350-371$.

Risek, B., Klier, F. G., and Gilula, N. B. (1994). Developmental regulation and structural organization of connexins in epidermal gap junctions. Dev. Biol. 164, 183-196.

Schuetze, S. M., and Goodenough, D. A. (1982). Dye transfer between cells of the embryonic chick lens becomes less sensitive to $\mathrm{CO} 2$ treatment with development. J. Cell Biol. 92, 694-705.

Segretain, D., and Falk, M. M. (2004). Regulation of connexin biosynthesis, assembly, gap junction formation, and removal. Biochim. Biophys. Acta 1662, 3-21.

Severs, N. J., Rothery, S., Dupont, E., Coppen, S. R., Yeh, H. I., Ko, Y. S., Matsushita, T., Kaba, R., and Halliday, D. (2001). Immunocytochemical analysis of connexin expression in the healthy and diseased cardiovascular system. Microsc. Res. Tech. 52, 301-322.

Shaw, R. M., Fay, A. J., Puthenveedu, M. A., von Zastrow, M., Jan, Y. N., and Jan, L. Y. (2007). Microtubule plus-end-tracking proteins target gap junctions directly from the cell interior to adherens junctions. Cell 128, 547-560.

Veiga, E., et al. (2007). Invasive and adherent bacterial pathogens co-Opt host clathrin for infection. Cell Host Microbe 2, 340-351.

Zampighi, G. A., Hall, J. E., Ehring, G. R., and Simon, S. A. (1989). The structural organization and protein composition of lens fiber junctions. J. Cell Biol. 108, 2255-2275. 